\title{
Le journal de bord en formation : une parole de travail
}

\section{Dominique Bucheton et Alain Decron}

\section{Q OpenEdition \\ 1 Journals}

Édition électronique

URL : http://journals.openedition.org/trema/1362

DOI : $10.4000 /$ trema. 1362

ISSN : 2107-0997

\section{Éditeur}

Faculté d'Éducation de l'université de Montpellier

\section{Édition imprimée}

Date de publication : 1 janvier 2003

Pagination : 21-42

ISSN : 1167-315X

\section{Référence électronique}

Dominique Bucheton et Alain Decron, «Le journal de bord en formation : une parole de travail », Tréma [En ligne], 20-21 | 2003, mis en ligne le 01 avril 2003, consulté le 19 avril 2019. URL : http:// journals.openedition.org/trema/1362 ; DOI : 10.4000/trema.1362

Ce document a été généré automatiquement le 19 avril 2019.

Trema 


\title{
Le journal de bord en formation : une parole de travail ${ }^{1}$
}

\author{
Dominique Bucheton et Alain Decron
}

1 Cet article se propose d'étudier la relation entre construction d'une identité professionnelle d'enseignant et élaborations langagières stratifiées dans plusieurs tâches d'écriture, au cours de l'année de PE2. Le portfolio regroupe les différents éléments d'évaluation d'un stagiaire au cours de sa deuxième année d'IUFM. Le Journal de Bord est le troisième et dernier élément intégré au portfolio. Il est élaboré au cours d'un stage en responsabilité et témoigne de différents moments d'écriture et de pensée par rapport à la mise en œuvre.

2 Ces deux dispositifs ont donc comme caractéristique principale d'obliger les stagiaires à remettre en chantier écriture et pensée lors de plusieurs strates langagières successives. Nous émettons l'hypothèse que ce processus de réécriture permet de mettre la pratique à distance pour l'éclairer grâce à des apports multiples qui ont besoin de temps pour s'organiser en une trame réflexive complexe.

\section{Une écriture par étapes dans une double visée formative et évaluative}

Depuis plusieurs années, à Perpignan, nous travaillons dans les classes et en formation sur le concept d'étapes intermédiaires de l'écriture pour favoriser les apprentissages et la construction du sujet écrivant.

Cette dynamique, mise en place, observée et analysée dans de nombreuses classes (de ZEP ou non), montre l'intérêt du temps et des strates successives d'écriture pour développer en synergie le « dire » et le « penser » chez les élèves. Les apprenants écrivent, réécrivent, et remettent donc en chantier le fond de leur texte grâce à une série d'interprétations successives nourries par de multiples apports (culturels, savants, privés, familiaux...) et interactions sociales tous azimuts, suscitées au sein de la classe.

Les écritures évoluent donc, s'épaississent, les points de vue se confrontent, changent, les enjeux se déplacent. Langage et pensée se construisent simultanément. 
4 Nous tentons actuellement une expérience sur le site IUFM de Perpignan, au niveau du Département de Français, qui devrait se généraliser aux autres disciplines. Les stagiaires voient leur module évalué grâce à un portfolio regroupant trois éléments d'évaluation répartis sur l'année et rattachés aux trois stages en responsabilité :

- Le premier élément est centré sur une observation des élèves.

- Le deuxième élément est centré sur une observation de l'enseignant.

- Le troisième élément, ou Journal de Bord (JdB) est centré sur l'analyse immédiate, puis distanciée d'une séquence d'apprentissages. Nous souhaitons ici voir le réinvestissement des éléments précédents sous la forme d'une observation fine de la co-activité maître - élèves.

Ces trois éléments d'évaluation s'appuient de façon forte sur des relevés langagiers dans les travaux d'élèves et leur analyse, ce qui évite aux stagiaires de se perdre dans des remarques ou commentaires uniquement issus du domaine comportemental.

Ce travail place donc stagiaires et élèves dans un rapport homothétique à l'écriture pour apprendre, penser et se construire.

"J'ai moi-même l'impression d'avoir été placée dans un processus de réécriture des séances à mener et du présent mémoire, divers apports théoriques et interactions sont venus alimenter ma réflexion personnelle et pourtant j'ai le sentiment qu'il $y$ aurait encore matière à réécrire!» (Émilie, PE2).

7 Ces quelques lignes d'Emilie, en conclusion de son mémoire professionnel, posent bien les enjeux de la réécriture à visée formative, ainsi que les liens qui nouent les différentes formes de cette écriture (cours, exposés, portfolio, mémoire, compte-rendu de parcours personnalisé).

\section{Les journaux de bord, dans le portfolio, sont une parole de travail}

8 Les enseignants de l'IUFM ont prescrit une tâche, il s'agit d'étudier comment les stagiaires s'en sont emparé pour entrer en activité (nous devrions plutôt écrire activités, tant les travaux rendus sont la marque d'une appropriation individuelle de la tâche). Cette étude permet d'améliorer le dispositif expérimental, de poursuivre les travaux sur le pouvoir réflexif de l'écriture en formation et d'explorer l'activité du sujet écrivant et pensant.

9 En quoi l'écriture - réécriture apporte-t-elle une nouvelle dimension à l'incorporation de savoirs professionnels? Quels instruments psychologiques ont participé à la découverte d'un sens ou à l'élaboration d'une signification? De quelle nature sont les savoirs professionnels construits?

Quelle identité professionnelle est en train de se bâtir? Une identité d'agent (qui reproduit), d'acteur (qui garde une large marge d'interprétation), d'auteur (qui invente sa propre pratique et réussit à la faire partager à ses pairs qui le reconnaissent comme auteur) ? (Decron A., 2001 et 2002).

11 Quels nouveaux espaces d'interprétation (Réthoré J., 1982) ouvre cette pratique langagière de formation? Chaque stagiaire est le premier interprète de ses hypothèses qu'il trame - ou non - avec la théorie, les cours, les conseils de formateurs, la réalité du terrain, ses répertoires privés liés à son histoire et ses affects. Comment négocie-t-il avec lui-même la prise en compte de cette somme de savoirs d'origines diverses, pour construire une identité professionnelle opératoire? 
12 Quelle est l'action de cette dynamique de réflexion sur la mise en œuvre à venir: meilleure vision des écarts, réajustements, plus grande part de l'improvisation, régulation et contrôle de la pratique pendant le temps de la classe?

\section{3. Éléments méthodologiques}

\subsection{Le corpus}

13 Les stagiaires professeurs d'école en deuxième année d'IUFM, à Perpignan et à Montpellier ont pour tâche de tenir un journal de bord dont la forme et le contenu sont cadrés par une directive donnée en début d'année scolaire. Ce document général est ensuite commenté en T.D. par les différents professeurs, ce qui donne lieu à des interprétations forcément différentes, mais conservant tout de même le «noyau dur » du dispositif, c'est-à-dire la prise de distance critique sur la pratique, l'apparition d'un point de vue singulier sur les savoirs enseignés, et aider à l'élaboration de savoirs professionnels en jouant sur un processus de réécritures et donc sur le temps.

14 Nous reproduisons ici ce qui était donné aux stagiaires en début d'année :

Troisième élément d'évaluation

Date limite de remise :groupes A : 25/2/02; groupes B : 28/4/02.

Rédaction : individuelle.

Objectif : pratique de l'écriture réflexive en formation.

Nature : On demande aux stagiaires de tenir un «carnet de bord»: prendre des notes quotidiennement, dans le but de répondre succinctement à la question suivante : «quels moments de la journée m'ont apparu intéressants pour ma propre formation en français, ou au contraire déstabilisants, et pour quelles raisons? Quelles questions sont posées, quelles réponses sont données?».

À la fin du stage, ce carnet de bord doit vous permettre de rédiger un bilan: qu'avez-vous appris sur l'enseignement du français au cours du stage?

15 Au cours de l'année, des consignes écrites plus précises sont venues compléter le premier schéma :

Consignes pour le journal de bord du stage en responsabilité

Le Journal de Bord est bien sûr un outil d'évaluation de la formation, mais avant tout un outil pour penser sa pratique professionnelle. Il n'est en tout cas pas un outil destiné à juger de votre mise en œuvre, ce sont les visites qui assurent cette évaluation là.

Partie observation et commentaires au jour le jour

Les sous-domaines du Français étant nombreux, vous devrez choisir un des secteurs de cet enseignement (langue, lecture, écriture, français dans les disciplines...) et vous y tenir. Justifiez votre choix dans le bilan final.

Une des compétences professionnelles que nous souhaitons développer est la capacité d'observer précisément et d'interpréter l'activité réelle des élèves (et non seulement leur comportement extérieur) et la manière dont le maître la perçoit et la régule. Nous vous demandons de choisir un ou deux élèves que vous suivez régulièrement et de justifier vos jugements à leur sujet.

Une des formes possibles pour le journal de bord est la suivante: une double page A4 + A4 parjour.

- Page de gauche: la copie du document utilisé pour la préparation, avec les annexes (supports utilisés, documents distribués...) éventuellement. Vous pouvez reprendre le travail fait pour votre "cahier-journal» ou vos préparations, mais nous n'imposons aucune contrainte pour cette partie. Il nous faut seulement savoir de quoi vous parlez.

- Page de droite en vis-à-vis : vos notes prises «à chaud ", jour après jour.

Nous vous demandons une analyse et pas seulement une description ou un récit. Cela 
suppose que vous vous interrogiez:

sur l'activité réelle du maître et celle des élèves. Nous vous demandons de vous interroger sur ce qu'ils disent, ce qu'ils écrivent, ce qu'ils comprennent, ce qu'ils lisent... et comment le maitre le perçoit et le régule;

- sur les problèmes que peuvent leur poser les tâches (consignes), notions, supports... et aussi sur le fait que parfois ils ne leur en posent pas assez... ;

- sur la suite que vous avez décidé de donner à ces observations, en termes de décisions concrètes.

Partie bilan finale rédigée a posteriori

Quelques jours après la fin du stage, vous reviendrez sur votre journal de bord:

1. Vous pourrez rajouter des commentaires ponctuellement en le relisant (utilisez une couleur différente, des Post It...)

2. Vous rédigerez un bilan/une synthèse générale (quelques pages) pour dégager ce que vous avez compris / appris au cours de ce stage, mais aussi sur quels points vous estimez devoir continuer à travailler (projet de formation à venir pour le reste de cette année et plus tard).

(D. Bucheton, J. Caillier, J.C. Chabanne, A. Decron, M. Parayre).

Nous avons lu, corrigé et étudié de nombreux journaux de bord et nous avons effectué une analyse plus poussée sur une cinquantaine d'entre eux pour observer comment le stagiaire, par son écriture, met en relation différents moments de son activité avant, pendant et après le cours, comment il interprète l'activité des élèves, comment il pense et régule sa propre activité d'écriture et la prévision d'une nouvelle mise en œuvre.

\subsection{Les limites de l'analyse des discours sur l'action}

Nous n'avons sous les yeux qu'un discours écrit, parlant d'un moment de classe. Ce discours est situé et évoque des actions situées elles aussi. L'accès à la réalité de la classe est donc particulièrement indirect. Nous travaillons donc sur des choix de présentation réalisés par l'auteur du discours (et de la pratique). Ces choix représentent de simples traces de l'activité réellement vécue, ils sont indiciaires non seulement de ce qui s'est passé, mais déjà d'un niveau d'interprétation - par le maître écrivant - de ce qu'il a vécu. Ce niveau d'interprétation nous éloigne de ce que l'on aurait perçu lors d'une visite de classe, par exemple, mais dans le même temps nous rapproche de la pensée de l'auteur, de l'organisation de ses propres savoirs, nous renseigne sur les tris qu'il opère pour rendre compte de son vécu professionnel.

Nous sommes donc, avec ces récits d'action, dans une tension entre le filtrage introduit par un point de vue singulier formaté, et les dimensions interprétatives et dialogiques du discours tenu, qui nous apprennent beaucoup sur la sélection des objets de pensée convoqués.

Bien entendu, ces multiples niveaux de filtres, d'interprétants, nous incitent à la prudence quant aux conclusions avancées.

«Le sens n'est pas réduit à la conscience qu'en ont les acteurs. Mais pas plus qu'à l'analyse des chercheurs. Chacun de par sa position en possède une partie. " (Pineau et Le Grand, 1993).

\section{De la tâche à l'activité}

La tâche est un travail imposé qu'il faut mener à son terme et qui vise à faire intégrer des savoirs, s'approprier une culture, tout en élaborant les cadres et les règles de la vie intellectuelle et sociale. À l'École, la tâche est co-effectuée, irriguée par une activité 
langagière qui se développe sur le plan cognitif, mais aussi en terme d'étayage et de régulation de l'action. de la tâche donnée. Ce mouvement unique, est la trace d'un sujet singulier qui trouve sa propre manière de répondre à la tâche, en dosant finement obéissance et transgression, dans la visée de retirer un bénéfice cognitif, psychologique, affectif du travail enjeu, et continuer ainsi à se construire.

\section{Une écriture qui se construit entre filtres, postures et univers discursifs}

\subsection{Les filtres de l'interprétation}

Le journal de bord est le produit d'une tâche prescrite et de choix subjectifs qui manifestent l'affirmation d'une singularité du rapport aux savoirs professionnels, le plus souvent tacites et que nous cherchons à mettre en évidence.

Nous constatons que les tâches conjointes du maître et des élèves sont vues au travers d'une certaine diversité de composantes de leur subjectivité que nous appellerons par commodité des filtres ${ }^{2}$ : ce sont aussi bien des savoirs, des doxa, des expériences, des discours, des contraintes, des valeurs.

Plusieurs filtres ont émergé de l'analyse des contenus des journaux de bord, ils correspondent à un regroupement des types de savoirs ou croyances convoqués par les stagiaires pour interpréter l'action pédagogique ou didactique.

Nous montrerons de quels filtres les stagiaires se servent pour parler des tâches, se les représenter et les évaluer.

\subsubsection{Le rapport à l'institution évaluatrice}

L'écriture du journal de bord est d'abord une tâche de formation demandée au stagiaire qui participe de son évaluation finale certificative. Ce premier filtre est présent chez tous, mais la valeur qui y est attachée diffère considérablement. Les journaux de bord montrent des traces très matérielles de ce rapport à l'évaluation, à l'image de soi que le stagiaire cherche à construire auprès des formateurs (cahiers recopiés au propre, manuscrits dactylographiés, retravaillés après coup ou au contraire travail bâclé).

"J'ai l'impression que ce journal de bord vous sert à juger notre pratique en classe. C'est un contrôle supplémentaire qui fait qu'on est évalué sans arrêt sur ce qui se passe dans la classe. " (Véronique, PE2).

\subsubsection{La mise en rapport avec les enseignements et discours en T.D. à l'IUFM}

Les discours didactiques de l'IUFM sont ici discutés, mis en perspective. L'alternance est dynamique et permet de penser la question du temps. 
"Quel retour donner aux enfants sur leur production? Plus que le produit fini, j'ai bien conscience que c'est l'évolution de l'enfant, de son texte qu'il faut prendre en compte (réflexion que nous avons menée en cours), mais j'ai le sentiment que je ne pourrai le mettre en place qu'au fur et à mesure, à force d'analyser les productions des élèves. » (Pascale, PE2).

\subsubsection{Le rapport à l'enseignant titulaire de la classe} résistance à un groupe. L'identité professionnelle qui se construit, et se tisse avec l'histoire personnelle du sujet et son système de valeurs, son éthique, permet au stagiaire de se positionner comme appartenant à un groupe, celui des enseignants, en tant que discipliné ou en tant que contestataire.

\subsubsection{Le rapport aux contraintes objectives de la classe et de l'école}

«Les parents se sont encore plaints auprès de la directrice au sujet des devoirs.» (Julie, PE2).

La signification de la tâche se construit par rapport à un contexte qui dépasse la seule classe et qui est en rapport avec les effectifs, l'hétérogénéité, la relation avec les parents, les collègues et la hiérarchie, les instances consultatives et décisionnelles qui composent le tissu éducatif.

\subsubsection{Le rapport aux Instructions Officielles et programmes et à leur évolution}

De manière assez surprenante pour nous, cette relation en tant que telle est assez rarement explicite. Elle existe parfois dans les textes de synthèse qui accompagnent le journal de bord et qui sont écrits a posteriori. Nous devons noter cependant que les journaux de bord produits pendant l'année 2001-2002 (ainsi que de nombreux mémoires de français) ont posé le problème de la prise en compte des nouveaux programmes: certaines séquences ont été clairement construites par référence aux propositions émanant de la Consultation sur les nouveaux programmes. Le plus souvent, ceci n'est pas exprimé explicitement dans l'écriture, mais arrive dans l'argumentation orale comme une évidence, un implicite partagé, une culture commune des groupes de stagiaires dont nous avons suivi le travail.

Il semble donc qu'au moment de la formation initiale, les stagiaires intègrent facilement de nouvelles données programmatiques. Nous pouvons faire l'hypothèse qu'étant encore dans une dynamique d'apprentissage, de nouveaux programmes sont une nouveauté parmi d'autres nouveautés. La pratique individuelle vécue n'est pas encore suffisamment prégnante pour freiner ou interdire l'ouverture vers de nouvelles directions.

30 Nous verrons apparaître ces réticences après quelques années de mise en œuvre qui auront permis à chacun de bâtir des routines professionnelles suffisamment confortables pour tenter l'évitement. De plus, le « petit nouveau de l'équipe » doit faire allégeance à 
l'esprit du groupe constitué pour être accepté et reconnu, ce qui mène plus souvent sur les chemins de la reproduction que sur ceux de l'invention ou de la novation.

Pendant cette deuxième année d'IUFM, les changements dans les programmes sont perçus comme un objet de savoir qualitativement identique aux autres objets de savoir rencontrés.

\subsubsection{L'ensemble des savoirs mobilisés pour comprendre, construits pour agir}

Se trouve ici convoqué autour de l'écriture, la réécriture, la révision, la correction, un ensemble flou de conceptions, doxa, de provenances diverses. On en trace principalement l'origine par le lexique employé par le stagiaire. Il renvoie :

- aux modèles pédagogiques diffusés par l'IUFM. En effet, la présence ou l'absence de mots tels que projet, activité de l'élève, sens pour l'élève, motivation, norme... est signifiante, ainsi que les formats de fiches de préparations en forme de grille d'analyse de la séance ${ }^{3}$.

- aux modèles didactiques (ici par exemple les modèles théoriques de la réécriture). Nous pouvons ainsi tracer les effets de la rencontre directe ou indirecte avec l'ouvrage phare en France du groupe d'Ecouen ${ }^{4}$, ou avec nos propres approches de la réécriture. Les mots chantier, critères, grille d'évaluation, épaississement du texte, étapes intermédiaires de l'écriture, sont pour certains et non pour d'autres, des passages obligés :

"J'ai choisi de ne pas proposer de commentaire «type» ou de 〈grille» d'écriture. Partir de leurs propositions, même si je n'obtiens pas tout ce que je souhaite, me paraît plus formateur.» (Martine, PE2).

"J'ai choisi de leur faire construire une grille. Toutefois je ne voulais retenir que trois ou quatre points pour ne pas perdre de vue l'essentiel et pour ne pas perdre les élèves. C'est pourquoi j'avais déjà sélectionné les critères qui seraient retenus en classe. Les élèves ont cité plusieurs points dont ceux que je souhaitais. Je n'ai retenu que ceux-là, puis j'ai distribué la grille toute faite aux élèves afin de gagner du temps. Je ne pense pas que le fait que les enfants n'aient pas complété eux-mêmes cette grille, les ait empêchés de se l'approprier [...] Cette séance de remédiation s'est bien déroulée et a permis à de nombreux élèves de comprendre leurs erreurs. Cependant je pense que j'aurais dû aller plus loin. Comme le montre le tableau des réussites, la moitié des enfants n'a toujours pas bien répondu à la consigne lors de la deuxième réécriture. En effet, la plupart manque d'imagination.» (Eva, PE2).

- aux modèles pratiques de la didactique : routines, théories implicites, pratiques rencontrées en stage de pratique accompagnée. Les dates des séances d'écriture et leur espacement, leur relation avec des séances de grammaire ou de lecture sont par exemple un indicateur fort du modèle pratique pris en compte (adaptations approchées des modèles en vogue, véhiculées par les manuels notamment).

- aux savoirs savants de la discipline, comme la narratologie, la syntaxe, la grammaire de texte ou de discours, pour n'en citer que quelques uns. Là encore, on trouve peu de références explicites à un champ de questions, l'implicite se référant le plus souvent aux problèmes de cohérence et de cohésion rencontrés dans des copies d'élèves et notamment à l'occasion de « suites de textes » problématiques.

\subsubsection{La mise en jeu de son expérience et de sa culture autour d'un projet}

Le stagiaire construit son identité professionnelle individuelle en rapport avec sa culture, ses propres champs d'intérêt, qui lui servent de sous-bassement au projet de formation pour lui-même et ses élèves : 
"Je commence mon stage, impatiente de mettre en place le projet que j'ai préparé. Je m'intéresse notamment par le biais de mon mémoire, à la littérature autre que de jeunesse, et à la façon dont je puis l'aborder en primaire. Pour cette classe de C.E.1., j'ai prévu de ne travailler que sur un livre: La gloire de mon père de Marcel Pagnol. J'espère pouvoir sensibiliser les élèves à l'humour et à l'atmosphère de ce roman. Pour cela, je pense travailler simultanément sur des extraits du livre et du film. J'amènerai ainsi les enfants à la production d'écrits visant à adapter des passages du roman pour les filmer. Les enfants seront écrivains et acteurs. " (Béatrice, PE2). sujet : variation.

\subsubsection{La mise en jeu de son identité naissante de professeur : le rapport à l'image de soi}

Le stagiaire est renvoyé à sa propre autorité, liée aux affects et donc à son histoire de

"Je suis une personne qui n'est jamais sûre d'elle-même.» (Nathalie, PE2).

et il explore ses propres espaces d'autorisation et d'invention :

"L'enseignante m'a recommandé de partir de l'adjectif qualificatif épithète pour présenter l'attribut et du groupe nominal prépositionnel : exemple, la belle fleur, la fleur est belle; le chien du voisin, le chien est au voisin. Attribut??? Ce dont j'ai fort douté, que je n'ai pas vérifié, mais je me promets de le faire incessamment. J'ai donc essayé de m'y prendre autrement, en me basant sur la grammaire que j'avais à ma disposition (du texte au mot) et sur les conseils cette fois, d'une autre connaissance enseignante. » (Catherine, PE2).

\subsubsection{Les caractéristiques linguistiques des écrits mis en œuvre}

Elles sont d'une extrême hétérogénéité : les journaux de bord revêtent parfois l'aspect d'un texte unique et uni. Ce sont alors des récits linéaires. Ou au contraire, nous nous trouvons devant des couches successives de textes plusieurs fois repris, commentés ou réécrits. Les genres de discours, les formes énonciatives, la précision du lexique de la discipline varient considérablement.

Nous allons maintenant analyser une saynète relative au travail de groupe, constituée de trois moments d'écriture tirés du Journal de Bord de Marina (PE2) et montrant cette

41 Moment de la préparation de la classe (cahier journal) :

« Recherche dans la bibliothèque : 4 groupes de niveaux hétérogènes

"Wakoo, livre faune des Pyrénées

- livre apporté par les enfants 


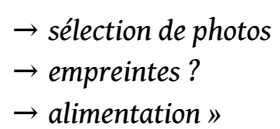

\section{(dans le JdB) :}

"Cette séance a mis en évidence les problèmes posés par le travail de groupe. D'une part, la collaboration ‘imposéè entre plusieurs élèves n'est pas facile. D'autre part l'hétérogénéité des âges au sein du groupe a perturbé les élèves. En effet, ils travaillent souvent en groupes, mais entre élèves d'une même section. Certains membres du groupe ignoraient et même repoussaient les petites sections. La mise au point, faite en demi-groupe classe a été constructive: elle a mis en évidence les rôles de chacun et en particulier celui des grands envers les petits. Elle s'est d'ailleurs élargie sceux qui comprennent aident ceux qui ne comprennent pas> (toutes sections confondues). Le travail de groupe s'est poursuivi plus sereinement. L'observation du comportement des élèves a montré que certains avaient bien pris conscience de leurs responsabilités et prenaient leur rôle très au sérieux. "

Nous constatons tout d'abord une importance quantitative croissante de l'écriture sur ce thème. Pourquoi ? La stagiaire, dans son cahier journal n'avait pas " pensé » le dispositif, le travail de groupe semble être un «allant de soi » simplement signalé en quatre mots dans la préparation de la classe.

La deuxième écriture nous montre un écart entre le prévu et le réalisé : il a fallu improviser pendant la mise en œuvre pour réguler le travail, redéfinir les rôles. Cette prise de notes révèle la prise de conscience de la maîtresse, confrontée à un obstacle imprévu. Nous sommes encore dans de la description d'action, car il faut gérer l'urgence et se concentrer sur la classe. Ces discours sur l'action ne sont pas l'action elle-même, mais ce qui intéresse le chercheur dans ce dispositif, c'est justement la focalisation du praticien sur tel ou tel objet de pensée, son interprétation de l'action pour aller vers la signification.

Le bilan montre, lui, une mise à distance tout autre. Les causes du "malaise » sont analysées (" collaboration imposée » et " hétérogénéité des âges»), tout en faisant référence à la connaissance que la stagiaire a des coutumes de la classe ( ils travaillent souvent en groupes mais entre élèves d'une même section»). Pour aller vers une remédiation, la stagiaire se déplace sur différents points de vue qu'elle a intégrés (celui de la titulaire, des élèves, des savoirs de l'IUFM). Elle construit ainsi son propre point de vue, résultant de ce tissage complexe, comme une tentative de transgression du « déjà mis en place " par la titulaire, non par simple désir d'opposition, mais dans le but de rechercher une adéquation entre une théorie sous-jacente et une mise en œuvre. En effet, Marina écrit plus loin : " La communication des informations au groupe classe a été un exercice très riche en échanges. Les enfants ont pu toucher du doigt la complémentarité de leurs recherches et ainsi la nécessité d'écouter les autres qui apportent des éléments nouveaux, la pièce manquante à notre réflexion. " La stagiaire généralise son improvisation et nous passons d'un concept d'étayage, non plus des grands vers les petits mais de ceux qui comprennent vers ceux qui ne comprennent pas. La suite du Journal de Bord montre un réinvestissement, dans la 
pratique de classe, de cette élaboration langagière. L'enseignante se positionne donc comme un sujet qui construit son identité professionnelle en croisant différentes expériences pratiques, savoirs théoriques, et peut-être même privés (notre réflexion semble renvoyer à une inclusion: la stagiaire parle également d'elle-même et de ses répertoires personnels).

\subsection{Des postures pour interpréter et agir}

L'enseignant, dans sa classe, ne peut rationaliser, programmer des compétences, et dans un même temps réguler et piloter des actions, toutes pertinentes (Bucheton D., 1997). S'il s'efforce d'anticiper, d'évaluer les objectifs de savoirs et de compétences à atteindre, il reste pourtant dans un clair-obscur cognitif qui procède de l'incertitude fondamentale et des tensions inhérentes à l'action éducative. Le chemin qui mène de l'enseignement prévu aux apprentissages réellement réalisés est semé d'improvisations, de digressions, d'écarts qui entraînent une série de décisions conscientes, ainsi que la mise en place de solutions/routines en acte pour gérer la classe.

Ces prises de décisions rapides sont le résultat d'un processus d'interprétation qui prend racine dans le contexte, les enjeux, les attentes, les partenaires réels. L'enseignant est un sujet plongé dans une histoire, tout comme ses élèves, et l'objet didactique en question, va donc créer, grâce à tous ces co-acteurs, une chaîne d'interprétations multiples pour élaborer ensemble de la signification. Ces mouvements de l'interprétation sont difficilement transmissibles et objectivables, puisque singuliers et contextualisés. Les gestes professionnels qui s'en dégagent sont forcément idiosyncrasiques, d'où l'importance du rôle des journaux de bord en particulier et de l'écriture en général, pour généraliser, argumenter une pratique, devenir auteur d'un discours pour soi et pour d'autres.

Si le concept de posture est aujourd'hui fréquemment utilisé, nous devons préciser ici l'acception que nous allons employer, en nous basant sur les travaux de Bautier et Bucheton (1997) relatifs à l'étude des postures d'écriture et Bucheton (1999) sur les postures de lecture.

"Les postures sont des schèmes préconstruits de pensée - langage - action pour traiter de problèmes langagiers et non langagiers, des manières de s'emparer d'une tâche ou d'un problème pour le résoudre, ou simplement le réaliser. La posture est relative à la tâche mais construite dans l'histoire scolaire sociale et personnelle du sujet. Les sujets disposent d'une ou de plusieurs postures pour négocier la tâche. Ils peuvent en changer au cours de la tâche selon le sens nouveau qu'ils lui attribuent. La posture est donc à la fois du côté du sujet dans un contexte donné, mais aussi de l'objet, ce qui en rend la saisie difficile et qui interdit tout (étiquetage) des sujets. " (Bucheton, 2001).

51 Les postures nous renseignent donc sur le rapport du sujet aux tâches, aux savoirs, au contexte, et en même temps son mode d'implication et son rapport au langage.

Nous postulons alors que la capacité à changer de posture en cours de tâche est un facteur du développement des compétences.

L'analyse des contenus des cahiers, corrélée à une analyse des pratiques langagières nous a permis de caractériser cinq grands types de postures, c'est-à-dire cinq manières de penser, interpréter les pratiques de classe. 


\subsubsection{Une posture scolaire} qui concernerait tout le monde, la classe est encore vécue comme un système duel enseignant - enseigné. L'analyse est réalisée le plus souvent en termes binaires de " réussi/pas réussi ». La tâche est transparente, sa compréhension est un « allant de soi ».

"Le questionnement portant sur les différentes productions est enrichissant. Pratiquement tous les élèves trouvent ce qui va ou ne va pas. Au contraire, la grille de relecture parait moins évidente. Ils ne comprennent pas forcément son utilité. » (Sandrine, PE2).

Les décisions prises dans l'action sont justifiées a posteriori, en particulier les suppressions de contenu à cause «d'un manque de temps» ou «d'une trop grande difficulté » et l'anticipation - justification des tâches suivantes.

"Séance beaucoup trop longue. Les enfants étaient très actifs lors de leur première écriture qui a duré trente minutes pour les premiers. Puisqu'ils ont été actifs, ils ont voulu presque tous lire. Devant l'enthousiasme, je n'ai pas voulu interrompre la séance: ils étaient tous dans l'activité, donc pourquoi arrêter? "(Élisabeth, PE2).

Nous constatons une forte hétérogénéité de discours (récit, commentaire, actes de langage de régulation adressés à soi-même). Des genres hétérogènes se superposent: plans de cours, relevés langagiers, commentaires, projets de planification future.

Tréma, 20-21 | 2003 
Il semble que cette posture corresponde à une représentation de la professionnalité abordée selon deux principes majeurs :

- 1. Le stagiaire évalue son action et les effets qu'elle produit au regard de modèles qui ne sont ni objectivés, ni interrogés. Il agit en fonction de doxa pédagogiques, didactiques, pratiques qui restent des savoirs flous, incertains, peu incorporés.

- 2. Le stagiaire pense sa mise en œuvre en fonction des discours et injonctions de l'enseignant titulaire (expert et connaisseur de la classe), sans aucune remise en question de ces « vérités immuables ». La pratique est donc vécue comme une obéissance à des règles qui ont été conçues par plus compétent que soi, par une instance supérieure de savoir sur la classe.

Cette posture, qui est fortement présente au cours des deux premières semaines chez presque tous les stagiaires, se caractérise par une absence d'hypothèses multiples sur les incidents ou écarts repérés. La gestion de la complexité n'apparaît pas encore, le stagiaire ne se considère pas vraiment comme un sujet auteur de sa didactique. Il se contente d'énoncer des généralités qui pour lui, ont force de lois.

\subsubsection{Posture d'observation d'élèves singuliers}

60 La question qui pilote cette posture est essentiellement : qu'ont-ils appris chacun ? Les aije suffisamment aidés?

Cette troisième posture intervient assez rarement en début de stage, même chez des stagiaires ayant déjà enseigné. Sans doute faut-il le temps de faire connaissance. Peutêtre aussi faut-il que la demande soit suffisamment claire pour que le discours devienne explicite. Auparavant, ce discours n'existait-il pas ou n'arrivait-il pas à la surface des journaux de bord? Les analyses que nous faisons pratiquer à nos stagiaires depuis quelques années commencent-elles à porter leurs fruits? Nous devons noter que si cette posture était très minoritaire, il y a deux ans encore (de l'ordre de $10 \%$ du corpus), elle devient actuellement très majoritaire (plus de $50 \%$ du corpus étudié). Nous noterons également que si les enseignants titulaires sont capables de commenter les apprentissages de chaque élève de manière individualisée, le discours tenu de façon informelle (lors des récréations par exemple) est très souvent globalisant («ils »). Ce discours, reçu sur le mode de la confidence initiatique (il se joue là l'acceptation du stagiaire dans un groupe social et professionnel qui le reconnait comme pair et lui livre les codes d'accès), reste probablement un modèle prégnant qu'il est difficile de remettre en question dans un premier temps.

Dans cette troisième posture, le stagiaire est donc capable d'identifier des groupes différenciés de résultats, ce qui l'oblige à prendre la mesure réelle de la tâche qu'il a donnée. Il prévoit donc des aides pour les élèves, des modifications et des adaptations de son projet. Il distingue les difficultés précises d'élèves singuliers sur des tâches singulières et cherche à les interpréter :

"Certains (Anthony, Jean-Baptiste) ont recopié des passages entiers; ne sachant pas trop quoi faire, ils sont voulu être certains d'écrire quelque chose de juste. » (Frédéric, PE2).

Les modèles pédagogiques sont alors interrogés, évalués, adaptés :

"La première remédiation (grille - modèle cognitif) a permis de résoudre de nombreux problèmes: ils ont compris ce que c'était qu'un dialogue et comment le présenter, mais cela n'a pas apporté de solutions à ceux qui manquaient d'idées, qui ne savaient pas quoi écrire, comme Julien. » (Frédéric, PE2). 
La stagiaire responsable de l'extrait ci-dessus essaie différentes formes d'aides, passe par des formes d'étayage entre pairs. Elle invente à partir des modèles connus. D'autres mettent en place du tutorat (très valorisé à l'IUFM de Perpignan).

Cette posture s'accompagne le plus souvent d'une forte prise en compte des contenus culturels des apprentissages. Ce contenu culturel est à la fois un cadre d'interprétation et un cadre de visée qui oriente les actions, les analyses, la mise en œuvre.

Nous voyons apparaître une visée politique du métier, comme chez Karine qui est touchée par les échecs de Julien et Estelle :

"Il est important que les élèves surtout en difficultés se sentent valorisés et qu'ils voient que leurs erreurs ne sont pas définitives. Ils ont droit à une autre chance. » (Paule, PE2).

\subsubsection{Posture identitaire (l'invention et l'affirmation de soi)}

La question organisatrice est: Quel professeur ai-je envie de devenir ? Qu'ai-je appris sur ma façon d'aborder la didactique du français?

Les réflexions explorent :

- La nature du projet didactique, les supports choisis, son ambition, ses conditions de faisabilité.

"Est-ce que je délire? La lecture au second degré de l'œeuvre n'a été perçue que par très peu d'élèves... Ai-je surestimé le niveau des élèves ou bien m'y suis-je mal prise pour leur faire saisir la portée symbolique de l'œuvre? Je ne sais pas. » (Audrey, PE2).

- L'espace d'autorisation, d'invention que laisse l'institution.

«Les pantoufles de l'institutrice me font mal aux pieds.» (Anne, PE2).

«En lecture, j'ai regretté de n'avoir disposé selon l'emploi du temps que d'une plage horaire très restreinte mais je reconnais que j'ai parfois du mal à sortir du cadre que l'on me propose car je suis malheureusement quelqu'un qui souffre d'un manque certain de confiance en soi... » (Fanny, PE2).

- Le projet d'autoformation dans des domaines notionnels ou pédagogiques perçus comme lacunaires.

- La relation contenus didactiques et autorité.

- L'écart entre la didactique - modèle et la réalité de sa propre pratique.

"J'ai le sentiment d'avoir été majoritairement transmissive. J'ai plaqué beaucoup de choses. Quand nous sommes à l'IUFM, en français notamment, je mesure la distance entre ce que je suis capable de faire à l'heure actuelle et la façon dont je dois essayer de m'y prendre en partant de ce que font les enfants, en posant des situations problèmes qui amènent à travailler une notion et à la justifier aux yeux des élèves. Je m'en sens très loin parfois et je suis assez découragée, mais même si le chantier est immense, c'est aussi une motivation pour moi. On n'a pas assez de temps et d'espace de dialogue pour revenir sur ce qu'on a fait... ” (Virginie, PE2).

Cette posture se trouve le plus souvent dans les écritures différées, réalisées après le stage, correspondant à un bilan général. Ces écritures sont encore très hétérogènes où le raconter, l'exposer et l'analyser se mêlent (Bronckart et al., 1985). Le questionnement permet de développer un point de vue singulier tout en adressant une critique voilée aux formateurs. Cette posture signerait un saut qualitatif, en s'autorisant à penser pour soi, se voir comme sujet enseignant.

\subsubsection{Posture de structuration de savoirs professionnels}

Si on reprend la formule selon laquelle apprendre c'est se poser des questions à un niveau différent ou supérieur, dans ce mode de penser les stagiaires interrogent leur propre 
capacité à penser ensemble et gérer les contenus d'enseignement prescrits, les modèles didactiques théoriques, des savoirs d'action qu'il viennent de se forger, la diversité des élèves réels insérés dans un contexte scolaire. Leur capacité d'interprétation, plongée dans leur propre culture privée et professionnelle, leur permettra plus ou moins efficacement de parvenir à décontextualiser l'expérience pour atteindre un niveau de généralisation, théorisation, conceptualisation. C'est cette opération qui aboutit à l'élaboration de savoirs professionnels persistants, c'est-à-dire susceptibles d'être réemployés dans un contexte différent de celui de leur apprentissage.

Cette posture peu fréquente en milieu d'année, marque une distance maximale par rapport à la pratique. Elle survient parfois pour rendre compte de moments de surprise qui indiquent un écart entre tonalité générale ou attentes et réalité.

«Pas de problèmes de discipline avec les élèves quand j'ai réussi à ce qu'ils soient vraiment concernés et en activité.» (Marc, PE2).

On la trouve par contre plus fréquemment dans les bilans de stage qui accompagnent et clôturent le journal de bord. Les stagiaires parlent alors :

- Des contenus didactiques ou des tâches fortement problématisées. Ils réfléchissent sur les nécessités et les finalités, les relations entre des domaines du français

- Du rôle de l'écriture du cahier de bord pour contrôler ses pratiques professionnelles et sa formation

"Face à divers problèmes rencontrés durant le stage, il m'est apparu utile de porter mes réflexions sur le cahier de bord, témoin de mes doutes et de l'évolution de ma pensée dans un certain nombre de domaines. Cet outil est assurément celui qui m'a permis avec le plus d'objectivité de mettre à distance mes pratiques d'enseignement. » (Thierry, PE2).

"Et je dois dire qu'écrire sur ce cahier de bord m'a permis une analyse plus fine, une réflexion plus systématique, chose que je n'aurais peut-être pas pris le temps de faire, en somme, il m'a permis de prendre un peu de recul par rapport à ma classe. Au fur et à mesure, je me suis aperçu que cette analyse, je la fesais presque sans m'en rendre compte tellement c'était devenu habituel, c'était devenu quasi systématique et me prenait beaucoup moins de temps, avec tout autant d'efficacité. » (Sabine, PE2).

- De la mise en relation du pédagogique, de l'observation des élèves et de leur propre projet de formation (postures 2, 3, 4).

Nous sommes, à ce niveau, face à une posture d'enseignant réflexif et donc capable de mettre en relation une grande variété de composantes et d'informations. Il pense, ajuste, régule sa classe dans cette complexité. Le discours est assez homogène et se situe dans l'exposer. Le texte est planifié, le vocabulaire didactique précis, ce qui concourt à montrer l'appartenance à la communauté des enseignants.

\subsection{Une parole qui circule dans trois Univers Discursifs}

Charles Sanders PEIRCE (1978) montre que l'on peut rendre compte de tout élément ou événement du monde grâce à trois catégories qu'il nomme priméité, secondéité et tiercéité.

En nous appuyant sur sa phénoménologie, nous pouvons suivre le développement de chaque discours, oral ou écrit, dans trois Univers Discursifs que nous appellerons l'Univers de l'Indéterminé, l'Univers de la Définition et l'Univers du Dé-nivellement (Decron, 2001). Ces trois Univers Discursifs sont respectivement liés aux trois catégories citées supra.

Quel est l'intérêt d'observer et de classer ainsi la parole produite? 
L'intérêt est à la fois cognitif et évaluatif. Cognitif, car le langage produit dans chaque Univers Discursif agit différemment sur la pensée en construction. Evaluatif, car la circulation au sein de ces trois Univers Discursifs nous renseigne sur les opérations mentales, le tissage d'informations, de savoirs incorporés ou extérieurs, la capacité à généraliser, conceptualiser.

L'Univers Discursif de l'Indéterminé témoigne d'une pensée en train de « muser » (Balat, 2000, pp. 23-27). Le stagiaire pense avec un objet de savoir ou une expérience concrète, mais un certain flou persiste.

Il ne donne pas d'indices. Habituellement, cette écriture ne serait pas destinée à être évaluée ou même montrée. Elle laisse libre cours à une pensée qui se déroule librement.

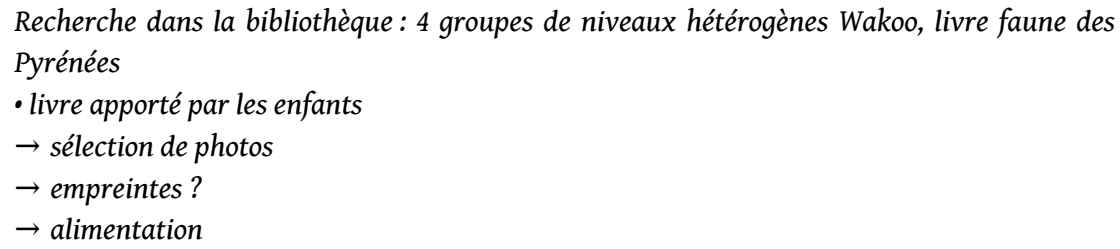

71 L'Univers Discursif de la Définition témoigne d'un point de vue singulier, d'un rapport indiciaire au monde. Nous savons que le stagiaire parle d'un objet et pas d'un autre. C'est un Univers qui porte les marques linguistiques de la précision car il s'agit de se confronter avec des existants : tel élève et pas tel autre, ce livre et pas tel autre.

"Cette séance a mis en évidence les problèmes posés par le travail de groupe. D'une part, la collaboration (imposée)) entre plusieurs élèves n'est pas facile. D'autre part l'hétérogénéité des âges au sein du groupe a perturbé les élèves. En effet, ils travaillent souvent en groupes, mais entre élèves d'une même section. Certains membres du groupe ignoraient et même repoussaient les petites sections. La mise au point, faite en demi-groupe classe a été constructive: elle a mis en évidence les rôles de chacun et en particulier celui des grands envers les petits. Elle s'est d'ailleurs élargie à cceux qui comprennent aident ceux qui ne comprennent pas> (toutes sections confondues). Le travail de groupe s'est poursuivi plus sereinement. L'observation du comportement des élèves a montré que certains avaient bien pris conscience de leurs responsabilités et prenaient leur rôle très au sérieux. " (Marion, PE2).

L'Univers Discursif du Dénivellement témoigne d'un saut qualitatif, d'une généralisation, pour aller vers l'énoncé d'un type ou d'une loi. Une marque linguistique typique est l'emploi de tournures favorisant l'apparition du mode infinitif, ce qui permet au sujet grammatical de ne pas être réalisé. Cette place vacante s'offre au lecteur ou à l'auditeur qui peut se positionner en tant que sujet possible du discours.

"Les discussions après les retours de stages ont été très intéressantes et bénéfiques. Le fait de discuter des différents problèmes rencontrés et d'essayer d'y apporter des solutions a renforcéle travail d'équipe. " (Virginie, PE2).

Notons ici qu'il faut être très vigilant à ne pas confondre émission de généralités et élaboration d'une généralisation. La généralité serait une pensée toute prête, que l'on fournit sans incorporation de savoirs, alors que la généralisation est la marque d'une induction ou d'une abduction. C'est pour éviter cet écueil que nous devons être attentifs à la nécessité de la circulation de la parole au sein des trois Univers Discursifs. D'où l'intérêt de faire produire des écritures pendant des moments différents de l'activité pédagogique, de débattre collectivement de ces écritures, de demander un travail sur des objets didactiques différents. Ce sont ces multiples éclairages, ces étapes intermédiaires $\mathrm{du}$ dire - penser, qui autorisent les explorations, associations, tissages propices à l'établissement de passerelles cognitives entre vécu, pratique de terrain, théories, 
domaine privé ou professionnel. La circulation dans les trois Univers Discursifs permet de garder en dialogue le feuilletage complexe constitutif du sujet. Celui-ci entre alors dans une dynamique qui lui permet de se déplacer pour modifier sans cesse son rapport aux savoirs, tout en gardant la conscience d'une permanence de lui-même dans les différents contextes vécus. Le sujet de l'apprentissage continue en effet à dire : «j'ai appris ceci » ou bien "je ne savais pas qu'on pouvait faire comme cela.» Le «je» est bien ici la marque linguistique de cette permanence qui relie dans un continuum celui qui ne savait pas encore à celui qui sait maintenant. La généralisation procède donc de l'exploration cognitivo-langagière de répertoires privés, de répertoires savants, d'une réalité de terrain, d'un contexte, d'une histoire. C'est à cette condition qu'elle promet des apprentissages et donc des changements d'habitudes, de postures.

Le professeur d'IUFM, dans un rapport homothétique au stagiaire professeur d'école, a donc la responsabilité de déceler les Univers Discursifs dans lesquels se développe le discours et de proposer des situations favorisant la circulation de chacun au sein de ces Univers.

L'expertise de l'enseignant consisterait alors à étayer la circulation dans les Univers Discursifs pour permettre à chaque enseigné de faire l'expérience d'un langage qui influe différemment sur le penser et l'agir, et rendre possible le changement de posture. Ceci ne peut se concevoir que dans un temps dévolu à la séquence d'apprentissage. Un temps pour penser avec, un temps pour élaborer son point de vue singulier et le confronter à d'autres, un temps pour généraliser, conceptualiser.

\section{Quelques pistes conclusives pour continuer le travail}

Nous voyons un grand nombre de PE2 se servir des différentes propositions d'écriture pour affiner leur recherche sur un sujet qui leur tient à cœur. Il est bien sûr difficile de savoir au travers de l'écriture si des savoirs professionnels sont incorporés, mais il est certain que l'intérêt de passer par de multiples strates langagières est devenu pour beaucoup une réalité. Nous avons obtenu dans la plupart des cas un engagement effectif dans ces étapes intermédiaires de la construction des savoirs, puisque de nombreux croisements ont existé entre modules de français, mémoires, parcours personnalisés. Des stagiaires ont écrit et publié un mini dossier dans le numéro 405 des Cahiers Pédagogiques pour relater et analyser les projets d'écriture-réécriture qu'ils ont mené sur le terrain. Nous voyons donc un réinvestissement et un transfert de compétences dans des pratiques professionnelles.

Même si de nombreux problèmes subsistent quant au pilotage par les stagiaires de leur tâche d'enseignement, il semble que la tenue de ce Journal de Bord joue un rôle d'outil dans la régulation de l'action. Il aide les stagiaires à identifier et objectiver les évènements perçus.

En conclusion, nous pourrions nous poser quelques questions centrales :

- Avons-nous introduit du doute et du flou grâce à cet outil?

Il semblerait que la réponse soit souvent positive. C'est bien dans ce flou que chacun peut

s'engager pour devenir auteur et non plus seulement agent ou acteur de son enseignement.

"Mes débuts furent difficiles [...] Il a fallu que j'apprenne à m'adapter à eux et non pas les pousser à s'adapter à moi. Une fois ce fait établi dans ma tête, je me suis trouvée plus 
détendue, plus attentive, plus à l'écoute de leurs progrès. » (Catherine, PE2). "Ce qui m'a le plus surprise, c'est le rapport à l'oral. » (Tamara, PE2).

- Avons-nous réussi à éveiller la dimension de singularité ?

Ce travail d'écriture à étapes multiples est une façon de renvoyer chacun à son propre rapport à l'écriture. Ce qui est travaillé, encore une fois, c'est la dimension d'auteur, dans sa singularité, dans son lien avec les programmes, les élèves, les parents, le reste de la communauté éducative.

"L'entrée dans l'activité est difficile. Les enfants n'ont pas l'habitude d'écrire et ont peur de se tromper [...] Le fait que j'écrive des mots de vocabulaire au tableau a été très bénéfique: certains élèves ont ouvert des pistes que d'autres ont réutilisées dans un contexte différent. " (Virginie, PE2).

- Avons-nous permis des généralisations, des conceptualisations, l'élaboration de règles ou lois pour prendre la classe en main?

Les Journaux de Bord foisonnent de constructions qui consistent à mettre à distance l'expérience singulière pour la généraliser. Beaucoup de cours de l'IUFM sont remis en question sous la lumière de la pratique et rebâtis pas à pas, théorisés à nouveau à partir du vécu. Nous sommes probablement là devant des marques d'incorporation de savoirs professionnels.

"L'élaboration de mes fiches de préparation a évolué au cours de l'année grâce aux outils mis à notre disposition. De plus, l'analyse des séances pratiquées est un travail très formateur et enrichissant qui m'a permis de prendre du recul par rapport à mes fiches et d'être ainsi plus à l'écoute des propositions des enfants. »(Virginie, PE2).

"J'ai réalisé une bonne séance d'un point de vue ‘ traditionnel 〉, mais je suis passée à côté de quelque chose de très important: La Mise en Réseau, la comparaison avec d'autres albums. La visite de l'IMF m'a ouvert les yeux. » (Caroline, PE2).

"Mais que fait-on de ces productions d'hypothèses? Comment exploiter ces écrits? [...] Ce genre d'exercice, en contradiction avec la production pour être lu, ne risque-t-il pas de décevoir les élèves?»(Tamara, PE2).

\section{BIBLIOGRAPHIE}

BALAT M. : Psychanalyse, logique, éveil de coma. Le musement du scribe. Paris, L'Harmattan, 2000, $252 \mathrm{p}$.

BRONCKART J.-P. et al. Le fonctionnement des discours : un modèle psychologique et une méthode d'analyse. Lausanne (Suisse), Delachaux et Niestlé, 1985.

BUCHETON D. et BAUTIER E. : Conduites d'écriture au CLG et Lycée professionnel. Versailles, CRDP Versailles, 1997.

BUCHETON D. : " Du côté des maîtres », in Actes du colloque : Pratiques enseignantes, activité de l'élève dans la classe de français, Bucheton et Chabanne (organisateurs), Lettre de la DFLM, № 21, 1997.

BUCHETON D. : «Les postures du lecteur », in La question de la littérature à l'école. Grenoble, CRDP (P. Demougins et J.-F. Massol éditeurs), 1999, 
BUCHETON D. : Langage, savoirs et subjectivité (Mémoire HDR présenté à l'Université P. Valéry, Montpellier III). Montpellier, 2001.

BUCHETON D. et CHABANNE J.-C. : L'enseignant interprète, Communication au colloque DFLM, Neuchâtel (Suisse), 2001.

CHABANNE J.-C. et BUCHETON D. : Parler et écrire pour penser, apprendre et se construire. L'écrit et l'oral réflexifs. Paris, PUF, 2002, $252 \mathrm{p}$.

DECRON A. et al. : « Écrire, c'est réécrire », in Cahiers Pédagogiques, № 405, Paris, CRAP, 2002, pp. $50-58$.

DECRON A. : L'élève Smahan : auteur d'une communauté sémiotique (Mémoire de DEA sous la direction de Joëlle Réthoré). Perpignan, Bibliothèque Universitaire, 2001, 201 p. DECRON A. : «L'élève auteur : un sujet à la source d'une communauté sémiotique ", in Sémiotique Peircienne : état des lieux (ouvrage collectif dirigé par Joëlle Réthoré et Tony Jappy). Perpignan, Presses Universitaires de Perpignan, 2002, p. 59-69.

PEIRCE C.-S. : Écrits sur le signe (traduction et commentaires de Gérard Deledalle). Paris, Seuil, 1978.

PINEAU G. et LE GRAND J.-L. : Les histoires de vie. Paris, P.U.F. (Coll. : Que sais-je ?), 1993.

RETHORE J. : « Lecture et interprétation. Une partition sémiotique des savoirs », in Sémiosis, No 27, Baden-Baden (Allemagne), Agis-Verlag, 1982, p. 32-38.

VYGOTSKI L. : Pensée et langage (trad. de Françoise Sève). Paris, La Dispute, 1997 (Éd. orig. : 1934).

\section{NOTES}

1. Avec la collaboration de J.C. Chabanne, Jacky Caillier et Marc Parayre. Equipe LIRDEF-ALFA, IUFM de Montpellier.

2. Ces filtres, dans d'autres cadres théoriques renvoient aux concepts : d'interprétant (PEIRCE), d'instruments psychologiques (VYGOTSKI), ou de préconstruits sociaux (BOURDIEU, BRONCKART).

3. ZEITLER A. : «Une conception située de l'alternance », in Questions de recherches en éducation action et identité. INRP CRF CNAM, 2001.

«C'est parce que la situation en institut de formation est saturée d'une norme de rationalité technique et d'une idéologie de la compétence démontrable ici et maintenant, que les étudiants organisent leurs actions autour d'une démonstration de forme pédagogique théâtralisée. » p. 105. 4. JOLIBERT J. : Former des enfants producteurs de textes. Paris, Hachette, 1988.

\section{RÉSUMÉS}

L'article étudie le rôle d'une écriture quotidienne sur la pratique des stagiaires PE2 pendant le stage en responsabilité. Il met en évidence l'émergence d'un certain nombre de "postures d'enseignement " qui très tôt différencient les stagiaires. Il met aussi en évidence comment 
diverses strates d'écriture, permettant un retour réflexif sur la pratique accompagnent la construction d'une identité professionnelle singulière.

This article studies the role plan, ed by daily written logbook entries upon the professional practice of elementary school student teachers during the "stage en responsabilité" internship period held in their second year of training. It reveals the emergence of a certain number of "teaching attitudes/standpoints" which very quickly set the trainees apart from each other. It also reveals how various levels of writing, from simple notes to well thought out texts, by allowing the trainees to reflect on their professional practice, help them build their individual professional identity.

\section{INDEX}

Mots-clés : écriture réflexive, posture d'enseignement, univers discursif, parole de travail, journal de bord

Keywords : reflective writing, teaching attitude/standpoint, environment of discourse, professional vocabulary, logbook

\section{AUTEURS}

\section{DOMINIQUE BUCHETON}

IUFM de Montpellier

\section{ALAIN DECRON}

IUFM de Montpellier 